\title{
Feasibility study of a randomised controlled trial to compare (deferred) androgen deprivation therapy and cryotherapy in men with localised radiation-recurrent prostate
}

\section{cancer}

M Salji 1,2, R Jones ${ }^{2,3,4}$, J Paull,4, F Birrell ${ }^{1}$, J Dixon-Hughes ${ }^{3,4}$, C Hutchison $^{3,4}$, T E B Johansen ${ }^{5}$, D Greene ${ }^{6}$, N Parr ${ }^{7}$ and $H$ Y Leung ${ }^{\star, 1,2,6,8}$ on behalf of the members of the Cryotherapy in Prostate Cancer (CROP) study team ${ }^{9}$

${ }^{1}$ Department of Urology, NHS Greater Glasgow and Clyde, Glasgow, UK; ${ }^{2}$ Institute of Cancer Sciences, University of Glasgow, Glasgow, UK; ${ }^{3}$ Cancer Research UK Glasgow Centre, NHS Greater Glasgow and Clyde, Glasgow, UK; ${ }^{4}$ CRUK Clinical Trials Unit, University of Glasgow, Glasgow, UK; ${ }^{5}$ Department of Urology, Oslo University Hospital, Oslo, Norway; ${ }^{6}$ Sunderland Royal Hospital, City Hospitals Sunderland, Sunderland, UK; ${ }^{7}$ Wirral University Teaching Hospital NHS Foundation Trust, Wirral, UK and ${ }^{8}$ Beatson Institute, Glasgow, UK

Background: Salvage therapeutic options for biochemical failure after primary radiation-based therapy include radical prostatectomy, cryoablation, high-intensity focused ultrasound (HIFU), brachytherapy (for post-EBRT patients) and androgen deprivation therapy (ADT). ADT and salvage prostate cryoablation (SPC) are two commonly considered treatment options for RRPC. However, there is an urgent need for high-quality clinical studies to support evidence-based decisions on treatment choice. Our study aims to determine the feasibility of randomising men with RRPC for treatment with ADT and SPC.

Methods: The randomised controlled trial (CROP) was developed, which incorporated protocols to assess parameters relating to cryotherapy procedures and provide training workshops for optimising patient recruitment. Analysis of data from the recruitment phase and patient questionnaires was performed.

Results: Over a period of 18 months, 39 patients were screened for eligibility. Overall 28 patients were offered entry into the trial, but only 7 agreed to randomisation. The majority reason for declining entry into the trial was an unwillingness to be randomised into the study. 'Having the chance of getting cryotherapy' was the major reason for accepting the trial. Despite difficulty in retrieving cryotherapy temperature parameters from prior cases, 9 of 11 cryotherapy centres progressed through the Cryotherapists Qualification Process (CQP) and were approved for recruiting into the CROP study.

Conclusions: Conveying equipoise between the two study arms for a salvage therapy was challenging. The use of delayed androgen therapy may have been seen as an inferior option. Future cohort studies into available salvage options (including prostate cryotherapy) for RRPC may be more acceptable to patients than randomisation within an RCT.

*Correspondence: Professor HY Leung; E-mail: h.leung@beatson.gla.ac.uk

${ }^{9}$ Members of the Cryotherapy in Prostate Cancer (CROP) study team are listed before References.

Received 11 February 2014; revised 9 May 2014; accepted 12 May 2014; published online 19 June 2014

(c) 2014 Cancer Research UK. All rights reserved 0007-0920/14 
Salvage therapeutic options for biochemical failure after primary radiation-based therapy (external beam radiotherapy, EBRT or brachytherapy) include radical prostatectomy, cryoablation, highintensity focused ultrasound (HIFU), brachytherapy (for postEBRT patients) and androgen deprivation therapy (ADT). Salvage radical prostatectomy (SRP) not only offers the highest cancerspecific survival of up to $83 \%$, but also has a high incidence of side effects with a continence rate of $39 \%$ and potency rate of $28 \%$ after 5 years in a prospective study of 100 SRPs (Stephenson et al, 2004; Chade et al, 2011). Hence, SRP is only recommended in highly selected patients with low comorbidity, organ-confined disease and favourable tumour parameters, namely PSA $<10 \mathrm{ng} \mathrm{ml}^{-1}$ and Gleason score $<7$ (Heidenreich et al, 2014).

Salvage prostate cryoablation (SPC) has an acceptable efficacy and toxicity profile (Ahmad et al, 2013). The latest update from the cryotherapy online data registry (COLD) of whole-gland prostate salvage cryotherapy for radiation-recurrent prostate cancer (RRPC) suggests a biochemical disease-free survival rate of $89 \%$, $73 \%$ and $66 \%$ at 1,2 and 3 years, respectively, without ADT (Spiess et al, 2013). SPC is typically associated with less morbidity than SRP as a result of the development of the third-generation cryotherapy technology and technical refinement in the procedure itself (Cytron et al, 2009), thus significantly reducing the incidence of fistula formation to $<2 \%$ and urinary incontinence to $\sim 10 \%$ in the latest multicentre studies (Finley and Belldegrun, 2011; Ahmad et al, 2013). In patients for whom local salvage therapy is not an option or not desired, a period of watchful waiting followed by
ADT is the current standard of care (Heidenreich et al, 2014). Although the absolute PSA levels considered appropriate to trigger ADT depend on multiple factors (PSA kinetics, tumour parameters and patient/clinician bias), a threshold of PSA level between 10 and $20 \mathrm{ng} \mathrm{ml}^{-1}$ is often accepted.

There is an urgent need for high-quality clinical studies to support evidence-based choice between SPC and ADT for patients with RRPC. The CROP (CRyOtherapy in Prostate cancer) study is a randomised controlled trial designed to compare (deferred) ADT alone and SPC for patients with recurrent prostate cancer after primary radical radiation therapy.

\section{PATIENTS AND METHODS}

CROP study design and patient eligibility. The CROP study is a randomised controlled trial of deferred ADT or upfront cryotherapy in men with localised RRPC designed to evaluate the efficacy and tolerability of SPC. The overall study design is illustrated in Figure 1 . To assess the feasibility of the entire study, an initial study period of 2 years was planned to adequately set up a suitable study network among participating centres (Supplementary Appendix 1) and to obtain accurate information on the likelihood of successful recruitment to the study. The primary study outcome measure was distant metastasis-free survival (DMFS; Supplementary Appendix 2) as assessed by CT of the abdomen and pelvis and isotopic bone scans (at least annually for up to 7 years, with extra scans

Trail scheme

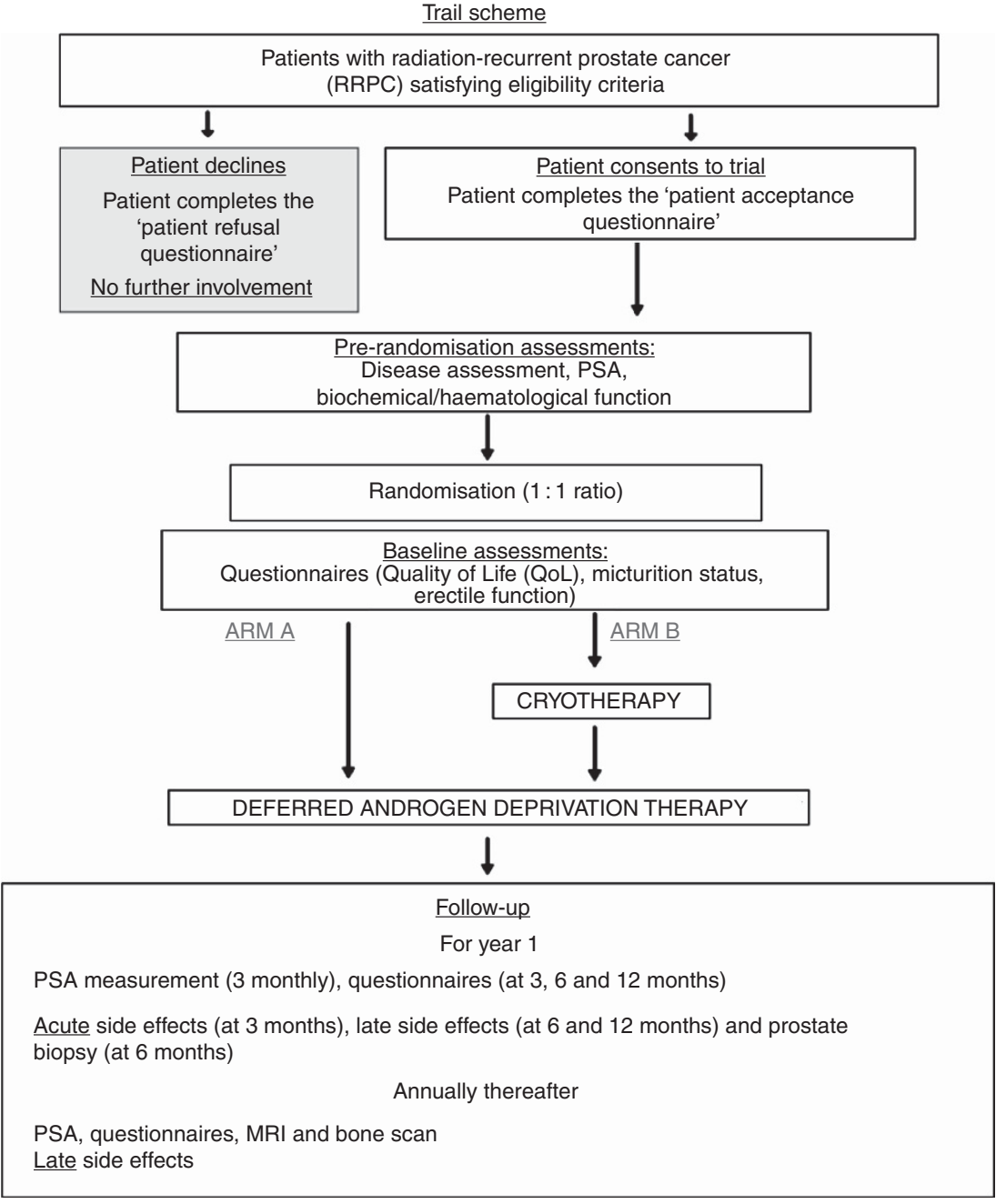

Figure 1. Trial Scheme. Schematic illustration of the CROP RCT trial design showing the patient pathway and timing of randomisation into deferred androgen deprivation therapy (arm A) and upfront whole-gland prostate cryotherapy (arm B). 
performed as indicated clinically). Secondary outcome measures can be categorised as following: (1) efficacy (biochemical disease control by serial prostate-specific antigen measurements and pathological response rate as assessed by prostatic biopsies at 6 months after SPC); (2) functional status of patients using validated questionnaires at the time of randomisation and at 3, 6 and 12 months, then annually thereafter: quality of life (EORTC QLQ-C30 and EQ-5D), micturition status (EORTC prostate cancer-specific module, PR-25) and erectile function (The International Index of Erectile Function, IIEF); (3) acute and late side effects of treatment assessed using NCI CTCAE v4.0; and (4) economic outcomes as the incremental cost per distant metastasis-free (DMF) year gained and quality-adjusted life year (QALY) gained from salvage cryotherapy in comparison to deferred androgen ablation.

Key inclusion criteria to the study include (Supplementary Appendix 3): (1) histologically confirmed relapsed prostate cancer following previous treatment with radiation therapy (either external beam or brachytherapy) for either organ-confined or non-metastatic locally advanced prostate cancer, namely $\mathrm{T}_{1-3 \mathrm{a}} \mathrm{N}_{0} \mathrm{M}_{0}$ disease; (2) life expectancy of at least 5 years; (3) clinical/radiological T1c-T3a; and (4) PSA level $\leqslant 20 \mathrm{ng} \mathrm{ml}^{-1}$.

Recruitment workshop. The recruitment workshop was an integral part of the CROP set-up and was based on evidence from the literature in maximising patient understanding for informed consent in order to ethically optimise recruitment rates. Key findings from the qualitative research substudy of a large on-going trial comparing different strategies for primary treatment of organconfined prostate cancer were incorporated (Donovan et al, 2002). The contents relevant to the CROP study include the following items: (1) overview of the CROP study and factors necessary for informed consent; (2) introduction of an information appointment that could be nurse led; (3) discussion of key issues such as clinical equipoise and how to present treatments equally, importance of an RCT in this area, discussion of randomisation as a reasonable method of treatment choice, order of presenting information, avoidance of mis-interpreted terms, multi-professional team working in information giving/consent, eliciting and challenging patients' views if at odds with the available evidence; (4) follow-up consent discussion and obtaining informed consent; (5) good and poor examples of consent in practice; and (6) how and when to administer the patient refusal or acceptance questionnaire.

The anticipated outcomes of the workshop were: (1) clinician recruiters (nurses, oncologists and surgeons) will accept clinical equipoise as it relates to the CROP study; (2) clinician recruiters will be confident about discussing the trial with patients, along with the need for the study, and will discuss randomisation as a reasonable method of treatment choice for the patient with localised RRPC; (3) recruitment rates should meet recruitment targets, with patients consenting to randomisation and accepting the treatment allocation; and (4) improved compliance with completion of the questionnaire for refusal of entry into the study.

Quality assurance of cryoablation procedure. The quality assurance process for individual cryotherapists forms another key component of the CROP study design. Participating cryotherapists were required to complete a Cryotherapists Qualification Process (CQP) before recruiting patients for randomisation within the CROP study. Data from three representative 'solo' cases were collected and reviewed by a panel chaired independently by Professor Truls Erik Johnasen (Denmark). Criteria being reviewed included (Supplementary Appendix 4): (1) effective use of transrectal ultrasound imaging to visualise the prostate and anterior rectal wall; (2) the planning and placement of appropriate ice 'needles' and temperature sensors; (3) evidence of continued monitoring of the iceball formed and temperature profile during the freezing phases; and (4) appropriate timing of the thaw cycles. As for the CQP, cryotherapy quality measures were continually collected prospectively within the study. In cases of concern, the CQP group would formally review the quality measures collected and the standard of the procedure for continued participation in the study.

\section{RESULTS}

Quality assurance of cryoablation procedure. Among the 11 participating centres previously performing prostate cryotherapy, 9 cryotherapists from 9 separate sites provided adequate data for formal assessment of prior 'solo' cases. The CQP assessed data on tumour characterisation (at initial presentation and at the time of relapse), details of previous radiotherapy, including total radiation dose and number of fractions for external beam radiotherapy, as well as duration and nature of ADT. In addition, the following technical parameters collected during the cryoablation procedure were assessed: the reported location of the ice needles, temperature profile and the dimension of iceballs at the end of freezing cycles. The CQP panel reviewed 33 cases during the CROP study, analysing these data from individual cryotherapists. Collectively, all prior cases submitted to CQP revealed expected satisfactory parameters for (i) patient selection (median time from primary treatment to RRPC 5 years, median Gleason score 7 and mean prostate volume $23 \mathrm{cc}$ ), and (ii) cryotherapy procedure (median freeze temperature of $-30^{\circ} \mathrm{C}$, median anterior rectal temperature $7^{\circ} \mathrm{C}$ and median iceball length $40 \mathrm{~mm}$ ). The CROP study team did experience difficulties in retrieving data from cryotherapy systems, particularly with the temperature profiles from prior cases. Nonetheless, it is worth noting that all cryotherapists who submitted adequate information received approval from the CQP panel.

Patient recruitment. Overall, four sites were opened to recruitment during the study period. The rest of the CQP-approved sites failed to secure support/approval from their respective host organisations. Three centres, namely the Beatson West of Scotland Cancer Centre, the Wirral University Hospital and Sunderland Royal Hospital, successfully recruited patients into the study. The formal CROP study was set to recruit 120 patients per year (540 in total over a period of 4.5 years). The 2 -year-long feasibility phase of the CROP trial was opened on 25 August 2011. Thirty-nine patients, or 26 per study-year, were screened for eligibility. Among them, seven patients agreed to randomisation and entered the study, while 21 patients declined participation. Two patients were deemed unsuitable due to risk of general anaesthesia, and two further patients were declined entry by practitioners for other reasons outside of study protocols. Seven of the screened patients (18\%) were considered ineligible when evaluated according to study protocol. After the feasibility study had been opened for a period of 18 months, a decision was made to close the study on the basis of failure to recruit.

Four of seven (57\%) patients recruited to the CROP feasibility study completed an acceptance questionnaire (Figure 2A; Supplementary Appendix 5). In the majority of these patients, multiple factors considered in the questionnaire were at play. All patients notably expressed a bias towards, or personal wish to receive, cryotherapy as their reason for acceptance (statement 1 in the questionnaire). For half of these patients, the chance of getting cryotherapy was the most important reason for agreeing to randomisation. All four patients considered the state of clinical equipoise to be important, that is, 'I did not know which treatment was best so it made sense to go into the trial', as a factor, but not as their most important reason, for acceptance.

Twenty-one screened patients were offered randomisation and entry into the CROP study, but declined to enter the study. Fourteen of these men (67\%) completed a declined entry in the CROP study questionnaire (Figure 2B; Supplementary Appendix 6). 
A

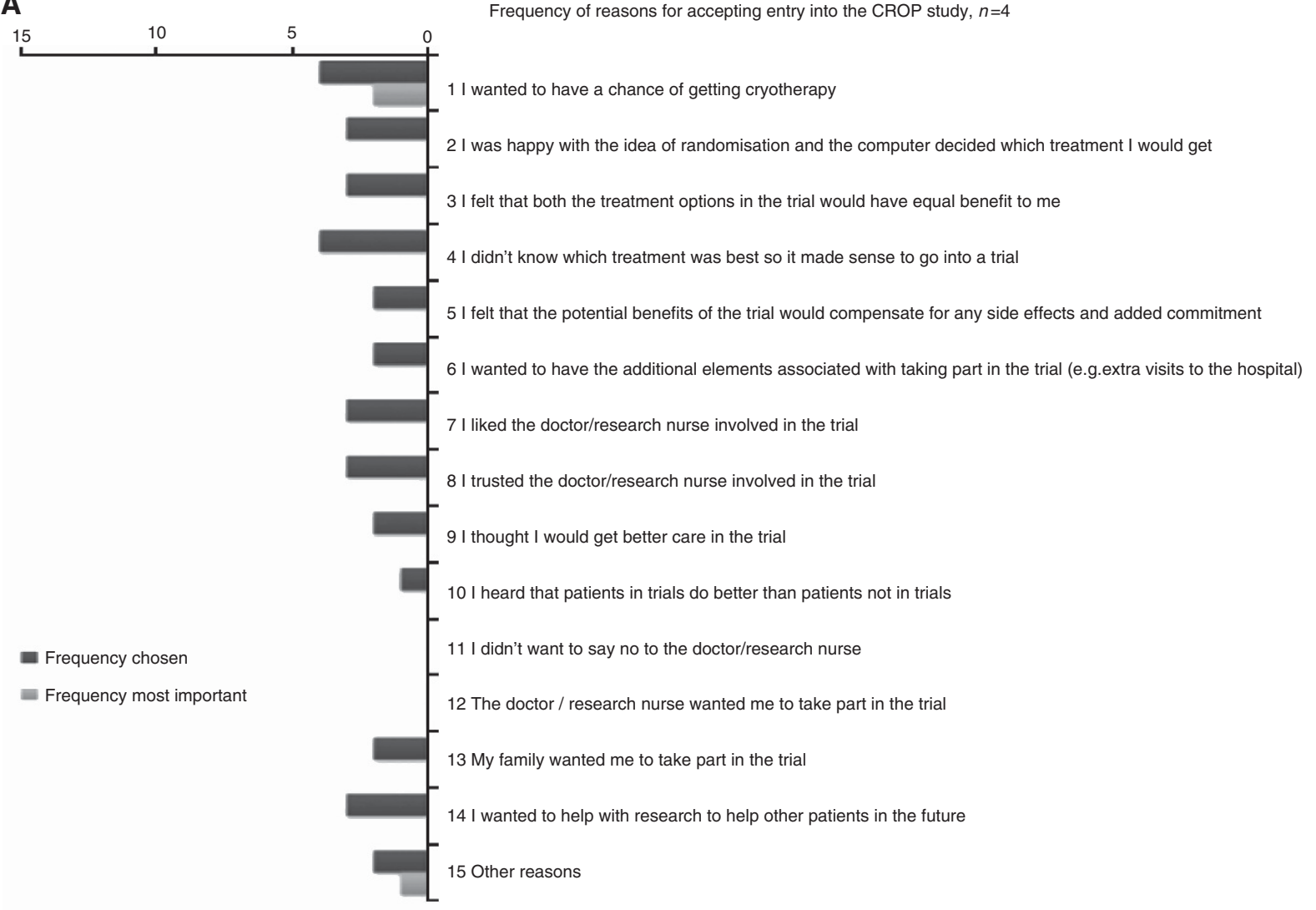

\section{B}

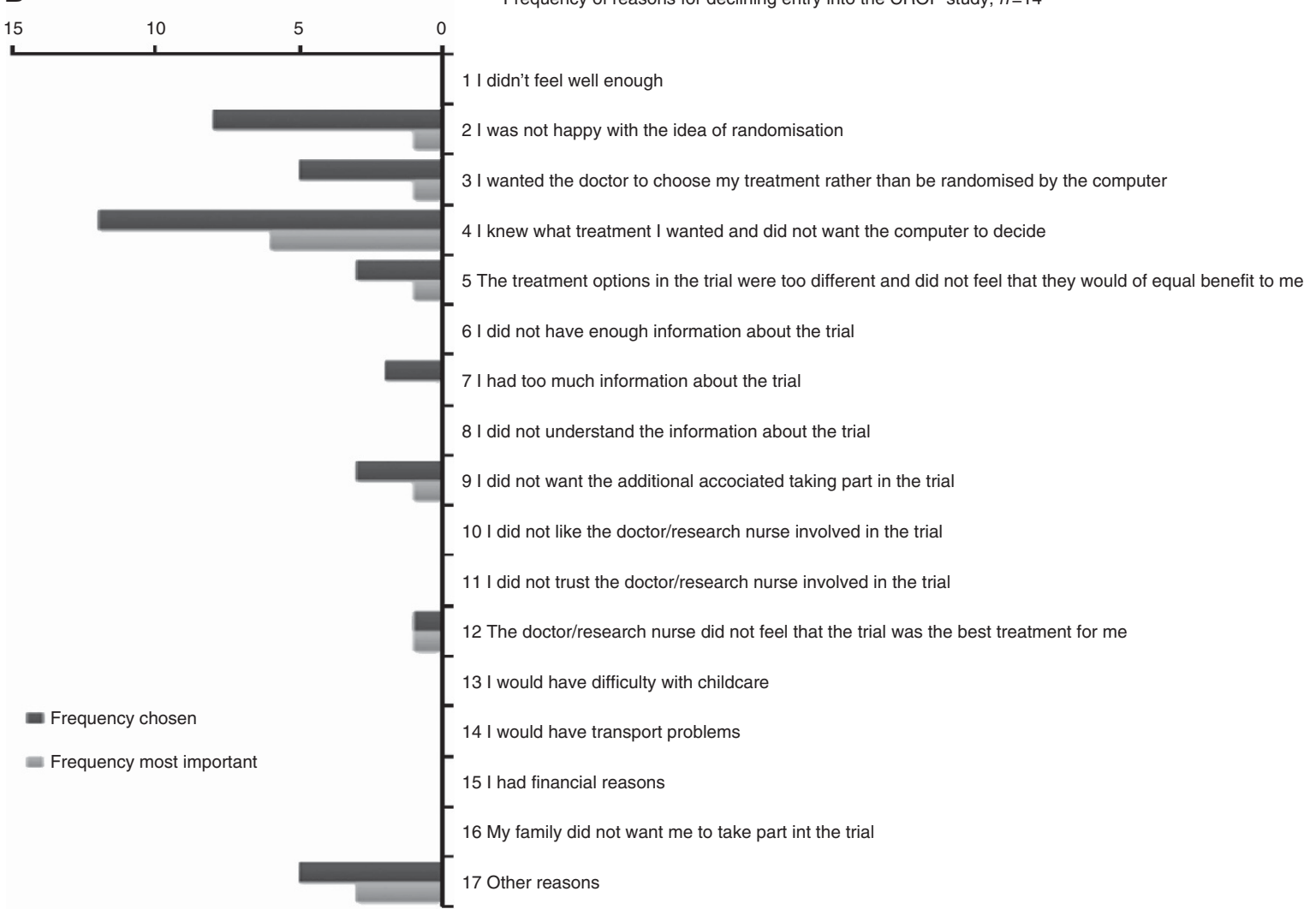

Figure 2. Histogram of frequency of reasons. (A) For accepting entry into the CROP study, and (B) for declining entry into the CROP study. Blue bars signify the number of patients in agreement with specific statements. For individual patients, the red bar signifies the statement being most important in their decision. The full colour version of this figure is available at British Journal of Cancer online. 
All 14 patients chose randomisation as a reason contributing to declining participation in the CROP study. Among half of them considered this to be the most important reason for declining. Statements relating to randomisation were overwhelmingly the reasons for patients to decline entry into the trial. Only 3 of 14 patients chose the additional statements in the declined entry questionnaire.

\section{DISCUSSION}

The feasibility phase of the CROP study was initiated to establish a system of quality assurance for prostate cryotherapy and to assess patient acceptability of randomisation into the CROP study. We encountered significant delay in opening recruiting centres due to a combination of difficulties in obtaining high-quality data for quality assurance assessment and securing approval from respective host organisations. Additional treatment costs related to cryotherapy and extra study scans required in our study are the contributing factors. Within the UK health system, it is clear that consensual support from both funding bodies for clinical trials and host organisations (Hospital Trusts and Primary Care Trusts, now Clinical Commissioning Groups) is critical.

Reasons for low recruitment into the study could be divided into patient and practitioner factors. Most patients with RRPC were not in a state of equipoise about the treatment options provided in the two arms of the CROP study. This made it difficult to present randomisation into either arm as equally acceptable to them. Our findings suggest that, for most patients, clinical equipoise between deferred ADT and upfront cryotherapy was not accepted. It is also worth noting that patients in the cryotherapy arm can subsequently cross over to receive ADT as clinically indicated. Furthermore, cryotherapy is also offered as a clinical service in parts of the UK, including Scotland as a salvage treatment. Patients in these areas may choose cryotherapy outside of the CROP study, which was a further obstacle to recruitment. Besides patient decision on participation, we report fewer eligible patients than expected presented for consideration. This is very much in keeping with a recent report (Tran et al, 2014), based on historic patient records, showing evidence for under-utilisation of local salvage therapies in patient with RRPC and good performance status, with as little as $2 \%$ going on to receive local salvage therapy.

From a population perspective, it is clear from the literature that evidence from randomised controlled trials remains lacking to show that cryotherapy would offer a survival benefit over expectant management with ADT (Alongi et al, 2013). Portraying equipoise on an individual basis, however, was clearly a major obstacle for patients to accept randomisation in this study. Although equipoise among recruiting clinicians and nurses was addressed at the investigators' training meetings, which included discussion surrounding carefully designed case studies, due to resource implications, clinical equipoise was not formally assessed/monitored within the study.

The CROP study design took a pragmatic approach to study the optimal treatment for an RRPC cohort by comparing (deferred) ADT and upfront cryotherapy by using metastasis-free survival as a primary end point. The ideal choice of end point would have been patient survival, but this would have required an unrealistically large number of patients to power as well as very long follow-up. However, the metastasis-free survival end point raised its own challenges due to resistance by host organisations to take on the burden of the extra follow-up imaging, even though the number of extra scans required per patient was low. The two arms within our study were based on the most commonly considered treatment options. Recently, a retrospective comparison using propensity-adjusted analysis between SPC and SRP has shown favourable results for SPC over SRP using data from Surveillance, Epidemiology, and End Results-Medicare linked data between 1992 and 2009 (Friedlander et al, 2014). Given the obvious limitation of such a retrospective analysis, a registration study in the future may provide robust data to inform treatment choice for patients with RRPC.

Even taking great care to optimise patient recruitment with a clinical equipoise approach, our feasibility study has demonstrated that we failed to apply the 'conventional' approach of RCT in this cohort of RRPC in comparing a non-surgical standard of care and cryotherapy as a surgical intervention. Our feasibility study may have benefited from qualitative analysis, as described in the ProtecT study previously, throughout its duration, but this would have had substantial resource and practical implications.

It can be argued that an RCT studying more than one salvage modality may increase the pool of patients eligible for recruitment. The consensus view of treatment options for patients with RRPC includes deferred ADT and cryotherapy, with SRP being recommended only in a very select group due to a high incidence of incontinence. Options such as HIFU or high-dose brachytherapy are performed only by a few centres. Hence, an alternative study design will still be subject to the same problems of randomisation and would dilute numbers in each of the study arms. In addition, if alternative local therapies such as prostatectomy and HIFU had been incorporated into the CROP study, it would have been necessary to put the relevant quality assurance in place for these highly specialised modalities, without the likelihood of a significant uplift in recruitment, as only a very small number of centres offer these options routinely.

Previous surgical RCTs have shown similar effects in comparing a surgical intervention with a conservative approach (Moynihan et al, 2012). In our patient cohort, the fact that our patients have recurrent cancer following previous radical treatment may further account for declining randomisation (Robinson et al, 2005). We therefore suggest that future cohort studies without randomisation may offer a viable option to obtain high-quality efficacy and treatment-toxicity data in a prospective manner.

Our findings also uncovered the difficulty in retrieving archival data from previous cryotherapy cases and that relevant data were not necessarily routinely captured clinically. We have recently demonstrated the potential usefulness of physical parameters such as temperature, gland length and iceball length in predicting patient micturition symptomatology (Ahmad et al, 2013). Hence, in future clinical study and routine practice, it will be useful for such data to be collected prospectively to facilitate meaningful corroboration between treatment parameters and patient outcome (for both efficacy and side effects).

\section{ACKNOWLEDGEMENTS}

We would like to thank the centres and patients who participated in this study. We would also like to thank Claudia Turley, Derek Jerome (Galil) and Roger Frais (Endocare) for their general support. We are grateful to Professor Jenny Donovan (University of Bristol) for sharing experience gained from the ProtecT study. This study was funded by the Cancer Research UK, trial number CRUK/ 10/048.

\section{CONFLICT OF INTEREST}

The authors declare no conflict of interest. 
MEMBERS OF THE CRYOTHERAPY IN PROSTATE CANCER (CROP) STUDY TEAM

Mohammed Ismail, David Gillatt (Bristol); Howard Kynaston, Malcom Mason (Cardiff); Mary Brown, Jennifer Petrie and Diann Taggart (Glasgow), Kirsteen Stuart, Sue Asterling (Sunderland); Gordon Muir (London); Frank Chinegwundoh (London); Alastair Henderson (Maidstone); Edwin Ho (Norwich); John Cumming (Southampton); Beverley Rogers, Gillian Riley and John Littler (Wirral)

\section{REFERENCES}

Ahmad I, Kalna G, Ismail M, Birrell F, Asterling S, McCartney E, Greene D, Davies J, Leung HY. (2013) Prostate gland lengths and iceball dimensions predict micturition functional outcome following salvage prostate cryotherapy in men with radiation recurrent prostate cancer. PloS One 8: e69243.

Alongi F, De Bari B, Campostrini F, Arcangeli S, Matei DV, Lopci E, Petralia G, Bellomi M, Chiti A, Magrini SM, Scorsetti M, Orecchia R, Jereczek-Fossa BA (2013) Salvage therapy of intraprostatic failure after radical external-beam radiotherapy for prostate cancer: a review. Crit Rev Oncol Hematol 88: 550-563.

Chade DC, Shariat SF, Cronin AM, Savage CJ, Karnes RJ, Blute ML, Briganti A, Montorsi F, van der Poel HG, Van Poppel H, Joniau S, Godoy G, Hurtado-Coll A, Gleave ME, Dall'Oglio M, Srougi M, Scardino PT, Eastham JA (2011) Salvage radical prostatectomy for radiation-recurrent prostate cancer: a multi-institutional collaboration. Eur Urol 60: 205-210.

Cytron S, Greene D, Witzsch U, Nylund P, Bjerklund Johansen TE (2009) Cryoablation of the prostate: technical recommendations. Prostate Cancer Prostatic Dis 12: 339-346.

Donovan J, Mills N, Smith M, Brindle L, Jacoby A, Peters T, Frankel S, Neal D, Hamdy F (2002) Quality improvement report: improving design and conduct of randomised trials by embedding them in qualitative research: ProtecT (prostate testing for cancer and treatment) study. Commentary: presenting unbiased information to patients can be difficult. $B M J 325$ : $766-770$.

Finley DS, Belldegrun AS (2011) Salvage cryotherapy for radiationrecurrent prostate cancer: outcomes and complications. Curr Urol Rep 12: 209-215.

Friedlander DF, Gu X, Prasad SM, Lipsitz SR, Nguyen PL, Trinh Q-D, Sun M, $\mathrm{Hu}$ JC (2014) Population-based comparative effectiveness of salvage radical prostatectomy vs cryotherapy. Urology 83: 653-657.

Heidenreich A, Bastian PJ, Bellmunt J, Bolla M, Joniau S, van der Kwast T, Mason M, Matveev V, Wiegel T, Zattoni F, Mottet N (2014) EAU guidelines on prostate cancer. Part 1: screening, diagnosis, and local treatment with curative intent-update 2013. Eur Urol 65: 124-137.

Moynihan C, Lewis R, Hall E, Jones E, Birtle A, Huddart R. SPARE Trial Management Group (2012) The Patient Deficit Model Overturned: a qualitative study of patients' perceptions of invitation to participate in a randomized controlled trial comparing selective bladder preservation against surgery in muscle invasive bladder cancer (SPARE, CRUK/07/ 011). Trials 13: 228.

Robinson EJ, Kerr CEP, Stevens AJ, Lilford RJ, Braunholtz DA, Edwards SJ, Beck SR, Rowley MG (2005) Lay public's understanding of equipoise and randomisation in randomised controlled trials. Health Technol Assess 9: 1-192, iii-iv.

Spiess PE, Levy DA, Pisters LL, Mouraviev V, Jones JS (2013) Outcomes of salvage prostate cryotherapy stratified by pre-treatment PSA: update from the COLD registry. World J Urol 31: 1321-1325.

Stephenson AJ, Scardino PT, Bianco Jr FJ, DiBlasio CJ, Fearn PA, Eastham JA (2004) Morbidity and functional outcomes of salvage radical prostatectomy for locally recurrent prostate cancer after radiation therapy. J Urol 172: 2239-2243.

Tran H, Kwok J, Pickles T, Tyldesley S, Black PC (2014) Underutilization of local salvage therapy after radiation therapy for prostate cancer. Urol Oncol: Semin Ori; e-pub ahead of print 11 March 2011; doi:10.1016/ j.urolonc.2013.12.014.

This work is published under the standard license to publish agreement. After 12 months the work will become freely available and the license terms will switch to a Creative Commons AttributionNonCommercial-Share Alike 3.0 Unported License.

Supplementary Information accompanies this paper on British Journal of Cancer website (http://www.nature.com/bjc) 